\section{Actividad antibacteriana in vitro de Croton lechleri sobre Streptococcus mutans}

\section{In vitro antibacterial activity of Croton lechleri on Streptococcus mutans}

\section{Resumen}

Objetivo: El propósito del presente estudio fue determinar la actividad antibacteriana de Croton lechleri (C. lechleri) sobre cepas de $S$. mutans ATCC 35668. Métodos: Se utilizó concentraciones al 50,75 y $100 \%$ de $C$. lechleri, como control positivo a la clorhexidina $0,12 \%$ y al agua destilada como control negativo. Se midieron los halos de inhibición producidos alrededor de discos embebidos con cada una de las concentraciones de $C$. lechleri sobre colonias de S. mutans sembradas en Agar Muller Hinton. Resultados: Las concentraciones al $75 \%$ y $100 \%$ de C. lechleri mostraron actividad antibacteriana in vitro sobre $S$. mutans, la concentración al 50\% no manifestó ninguna actividad. La clorhexidina al $0,12 \%$ mostró mayor actividad antibacteriana in vitro que las concentraciones de $C$. lechleri en los grupos de estudio evaluados. Conclusiones: Croton lechleri puede ser una alternativa natural, accesible y de bajo costo para métodos de prevención en caries dental al utilizarse al 75 y $100 \%$.

Palabras clave: Antibacterianos; Croton; Streptococcus mutans.

\section{Artículo Original}

Iván André Avilés Hidalgo 1,a , Marina Antonia Dona Vidale ${ }^{1, b}$, Christian Andrés Cabezas Abad $^{1, a}$, Cinthya María Quisiguiña Salem ${ }^{1, a}$

${ }^{1}$ Universidad Central del Ecuador, Facultad de Odontología. Quito, Ecuador.

a Odontólogo

${ }^{b}$ Especialista en Periodoncia

Correspondencia:

Iván André Avilés Hidalgo

Correo electrónico: livingtheglory@gmail.com

Carcelén, Urbanización "Los Mastodontes" 3ra transversal, Quito, Ecuador.

\section{Coautores: \\ Marina Antonia Dona Vidale marinadona perio@hotmail.com Christian Andrés Cabezas Abad ancabezas94@gmail.com Cinthya María Quisiguiña Salem chopi_cinthy@hotmail.com}

Conflicto de intereses: Los autores declaran no tener conflictos de interés.

Fuente de financiamiento: Autofinanciado

Fecha de recepción: 19/03/18

Fecha de aceptación: 09/07/18

\begin{abstract}
Objective: The purpose of this study was to determine the antibacterial activity of Croton lechleri (C. lechleri) on strains of S. mutans ATCC 35668. Methods: It was used 50\%, $75 \%$ and $100 \%$ concentrations of C. lechleri, and chlorhexidine $(0.12 \%)$ as a positive control and distilled water as a negative control. It was measured the inhibition halos produced around imbibed discs with each of the concentrations of $C$. lechleri on colonies of S. mutans which were seeded in Muller Hinton Agar. Results: 75\% and 100\% of C. lechleri concentration showed antibacterial activity in vitro over S. mutans, 50\% concentration showed no activity. $0.12 \%$ chlorhexidine concentration showed a higher antibacterial activity in vitro than all the concentrations of $C$. lechleri groups evaluated. Conclusions: Croton lechleri can be a natural, accessible and low-cost alternative for prevention methods in dental caries when used at $75 \%$ and $100 \%$.
\end{abstract}

Keywords: Anti-Bacterial agents; Croton; Streptococcus mutans. 


\section{Introducción}

La Organización Mundial de la Salud (OMS) calcula que en el mundo alrededor de cinco mil millones de personas han sufrido de caries dental, es decir, casi la totalidad de la población mundial ha sido afectada ${ }^{1-3}$. En Ecuador la caries dental y la enfermedad periodontal son las alteraciones bucales más prevalentes y de mayor demanda de atención sanitaria, convirtiéndose su prevención y tratamiento en un reto para los profesionales de la salud ${ }^{4,5}$.

Se describe a la caries dental como una alteración multifactorial, que se desarrolla por la interacción entre un huésped susceptible, dieta rica en hidratos de carbono y bacterias del biofilm dental durante un periodo de tiempo. Su evolución es dinámica y se da por la pérdida del mineral de la superficie dental como consecuencia de la acción de ácidos, productos del metabolismo bacteriano, sobre el diente pudiendo formar una lesión irreversible ${ }^{6,7}$.

El Streptococcus mutans (S. mutans) sintetiza polisacáridos extracelulares de la sacarosa a través de las enzimas glucosiltransferasa (GTF) y fructosiltrasferasa (FTF), las cuales son capaces de producir glucano y fructano respectivamente, y le facilitan la adherencia y nutrición al microorganismo ${ }^{8}$. La superficie dental es un hábitat natural para el $S$. mutans y el sistema de quorum sensing del biofilm facilita su proliferación y dominio de la bacteria en esta estructura ${ }^{9}, 10$.

Es claro que el tratamiento no es por sí mismo la solución para combatir el problema de la caries dental. La implantación de métodos preventivos en el primer nivel de atención en salud permitirá disminuir la prevalencia de dicha enfermedad ${ }^{11,12}$. Por tanto, es evidente la necesidad del uso de antimicrobianos locales, a base de productos naturales o convencionales, que actúen disminuyendo la proliferación bacteriana, eliminando el biofilm ya formado y alterando el desarrollo del mismo ${ }^{13}$.

Croton lechleri (C. lechleri) es una planta originaria de la Amazonía Sudamericana que produce una resina denominada sangre de drago, por su gran parecido con la sangre humana. Diversos estudios actuales han demostrado sus propiedades medicinales, cicatrizantes y antivirales ${ }^{14-16}$. Según Cayo la sangre de drago tiene acción inhibitoria sobre cepas de $S$. mutans, en un análisis realizado in vitro ${ }^{17}$, además, actúa inhibiendo el crecimiento del Helicobacter pylori en concentraciones elevadas ${ }^{18}$.

Dentro de sus principios activos podemos mencionar metabolitos secundarios pertenecientes a diferentes grupos: fenoles, terpenoides, alcaloides, leptinas, polipéptidos, tales como: la taspina que actúa activamente en la cicatrización de heridas. La proantocianidina SP-303 con su acción antiviral y ciertos compuestos fenólicos, ácido clorequínico y coberinas A y B que cumplen funciones antimicrobianas ${ }^{19}$.

Con estos antecedentes, el presente estudio buscó determinar si la sangre de drago ( $C$. lechleri) produce una actividad antibacteriana sobre el crecimiento in vitro de $S$. mutans, buscando impulsar el uso de este agente natural de origen ecuatoriano como un coadyuvante alternativo, asequible y accesible, en la prevención de la caries dental en la población que tiene dificultad para acceder a los servicios de atención sanitaria en nuestro país.

\section{Métodos}

El estudio fue de tipo experimental, in vitro; realizado en los laboratorios de botánica y microbiología de la Universidad Central del Ecuador en condiciones controladas, sin compromiso con seres vivos, se trabajó con cepas biológicas adquiridas mediante importación directa, que crecen en el laboratorio, por tanto, no fue necesario un consentimiento informado. Todos los aspectos éticos, jurídicos, metodológicos y el procedimiento a desarrollar fueron previamente analizados y aprobados por el Comité de Ética de la Universidad Central del Ecuador.

La sangre de drago se obtuvo en estado puro, directamente de los árboles de $C$. lechleri desarrollados en suelos aluviales a una altura aproximada de 800 m.s.n.m. en los bosques tropicales húmedos de la provincia de Morona Santiago en la Amazonía ecuatoriana, realizando una sección longitudinal en la corteza del tronco de donde brota el látex al exterior. Posteriormente se procedió a la dilución del látex de $C$. lechleri, para lo cual se preparó $10 \mathrm{ml}$ en concentraciones de 50\%, 75\% y $100 \%$ respectivamente y que fueron irradiadas para evitar cualquier tipo de contaminación.

El medio agar Mueller Hinton fue utilizado para realizar pruebas de sensibilidad debido al buen desarrollo que presentan las bacterias y la amplia evidencia que lo avala. La cepa de S. mutans ATCC 35668 fue adquirida mediante la empresa importadora Medibac, en estado liofilizado y almacenada a una temperatura de $5{ }^{\circ} \mathrm{C}$ de acuerdo a la recomendación del fabricante previo a su activación.

La activación de la cepa fue realizada en medio de cultivo Tripticasa Soya Agar (TSA) y fueron incubadas por 24 horas a $35^{\circ} \mathrm{C}$ siguiendo el protocolo recomendado por el fabricante. Se realizó la prueba de tinción Gram la cual resultó positiva y la prueba de la catalasa que obtuvo resultado negativo descartando contaminación. La actividad antibacteriana se comprobó siguiendo el protocolo establecido por la CLSI en su documento M02-A12 según el método de difusión en disco de Kirby-Bauer ${ }^{20}$, como se describe a continuación.

1. Estandarización. Para estandarizar la densidad del inóculo para realizar la prueba de sensibilidad se preparó la suspensión en Tripticasa Soya Caldo (TSC) de forma que presente la misma turbidez que la escala 0,5 McFarland, la suspensión bacteriana tuvo aproximadamente $1,5 \times 10^{8} \mathrm{UFC} / \mathrm{ml}$ de $S$. mutans ATCC 35668.

2. Inoculación. La siembra de la bacteria se realizó durante los 15 minutos posteriores a la estandarización con un hisopo estéril, el cual fue sumergido en el tubo de ensayo con la muestra y presionado contra la pared del mismo para remover los excesos. 
El experimento se realizó en duplicado realizando la siembra en tres direcciones diferentes para lograr distribuir la bacteria en toda la superficie del agar.

3. Colocación de discos. Los discos de papel fueron embebidos con las diluciones de sangre de drago $(50,75$ y $100 \%)$ mediante una pipeta con una cantidad $20 \mu \mathrm{L}$ en cada disco. Además, se realizó el control positivo con clorhexidina al $0,12 \%$ y el control negativo con agua destilada en cada placa Petri.

4. Incubación. Finalmente se trasladó a una jarra GasPak de incubación que brinda condiciones de anaerobiosis para el desarrollo bacteriano. Una vez colocadas las placas Petri se cierra la jarra GasPak, para dar condiciones de anaerobiosis a $35^{\circ} \mathrm{C}$ y CO 2 al $5 \%$; y así lograr una adecuada incubación de los microorganismos.

5. Recolección de resultados. Los resultados obtenidos fueron leídos mediante las mediciones de los halos de inhibición a las 48 horas de incubación, con la ayuda de una regla graduada en milímetros y un equipo Quebec (contador de colonias) para pro- porcionar luz y lograr una mejor observación de la actividad antibacteriana.

Cabe indicar que se realizaron 15 repeticiones de cada una de las muestras y todo el procedimiento se desarrolló en una cámara de flujo laminar clase II e instrumental estéril suministrado por el laboratorio. Una vez terminada la fase experimental, se eliminaron los desechos producidos durante el estudio, procedimiento realizado de acuerdo al protocolo de manejo de desechos establecido por el laboratorio de Análisis Clínico y Bacteriológico de la Facultad de Química de la Universidad Central del Ecuador.

\section{Resultados}

Se estudió la inhibición de crecimiento bacteriano que produce $C$. lechleri sobre la cepa de $S$. mutans, aplicando la prueba de sensibilidad de difusión en disco de Kirby - Bauer ${ }^{20}$. Los datos se obtuvieron con una regla milimetrada midiendo los halos de inhibición producidos por la actividad de la sangre de drago embebida en los discos sobre el cultivo bacteriano como se puede ver en la Figura 1.
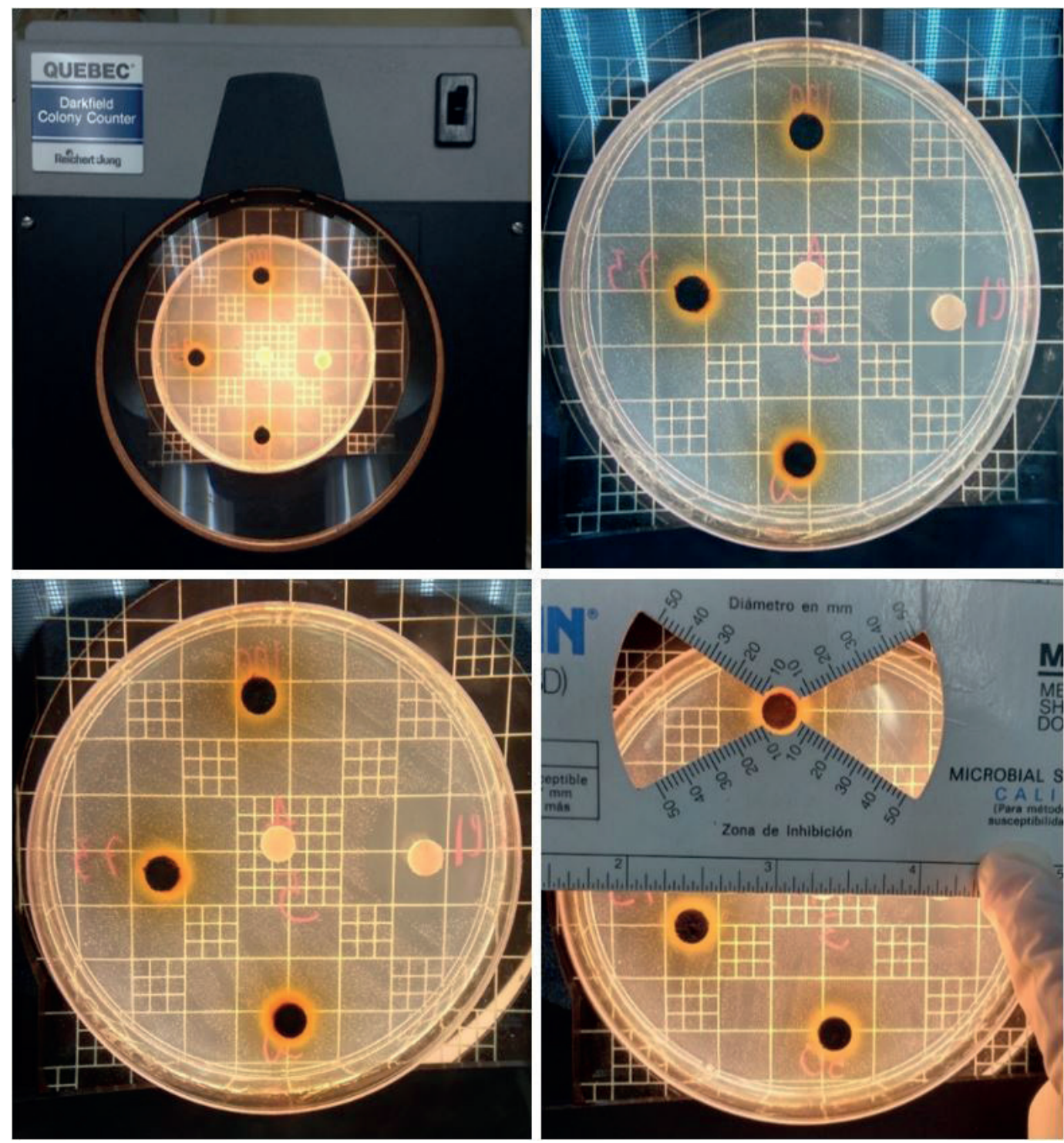

Figura 1. Medición de los halos inhibitorios 
Una vez recolectados los datos de la medición de los halos de inhibición producidos por C. lechleri sobre $S$. mutans se realizó el análisis estadístico de los mismos, utilizando el programa de análisis estadístico SPSS 20,0., cuyos resultados los podemos observar en la Tabla 1.

Como parte del análisis estadístico se observó que los halos inhibitorios presentan una mediana de $8,20 \mathrm{~mm}$ y 10,8 mm para las diluciones de sangre de drago al 75\% y $100 \%$ respectivamente, en el grupo control positivo con clorhexidina una mediana de 23,07 mm. En la dilución al 50\% y en el grupo control negativo con agua destilada no se produjo efecto alguno, tal como se encuentra expresado en la Figura 2.

La prueba estadística de comparación por pares de Mann-Whitney determinó que la clorhexidina al 0,12\% produjo una actividad antibacteriana mayor que C. lechleri. Sin embargo, cabe mencionar que los halos de inhibición producidos por el látex, excepto la concentración al 50\%, son aceptables para tenerlo en cuenta como agente antimicrobiano accesible y asequible frente al S. mutans.

\section{Discusión}

La odontología contemporánea tiene como premisa utilizar medidas que permitan prevenir la caries dental y las enfermedades periodontales, patologías bucales más prevalentes en el mundo. Son cada vez mayores las sustancias, naturales o sintéticas, estudiadas para inhibir la acción del S. mutans, microorganismo que al interactuar con otros factores de riesgo puede desarrollar caries dental.

La investigación evaluó la existencia de una actividad antibacteriana de $C$. lechleri sobre cepas de $S$. mutans ATCC 35668 mediante la prueba de difusión en disco de Kirby - Bauer, luego de analizar los resultados se demostró la actividad antibacteriana producida por el látex del C. lechleri. Además, dependiendo de la concentración aplicada en el estudio, se encontraron variaciones significativas en la actividad producida (halo inhibitorio). Así lo demuestran estudios realizados por Corrales et al. ${ }^{15}$, y León et al. ${ }^{21}$, quienes en sus investigaciones in vitro, concluyen que la sangre de drago C. lechleri tiene una actividad antibacteriana sobre cepas de microorganismos Gram positivos anaerobios facultativos presen-

Tabla 1. Análisis descriptivo de los resultados

\begin{tabular}{|c|c|c|c|c|c|c|c|c|}
\hline \multirow{2}{*}{ MEDIDAS } & \multirow{2}{*}{$\mathbf{N}$} & \multirow{2}{*}{ 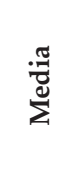 } & \multirow{2}{*}{ 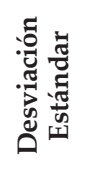 } & \multirow{2}{*}{ పี } & \multicolumn{2}{|c|}{$\begin{array}{l}95 \% \text { del intervalo de } \\
\text { confianza para la media }\end{array}$} & \multirow{2}{*}{$\underset{\Xi}{\stackrel{\Xi}{\Xi J}}$} & \multirow{2}{*}{ 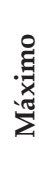 } \\
\hline & & & & & $\begin{array}{l}\text { Límite } \\
\text { inferior }\end{array}$ & $\begin{array}{l}\text { Límite } \\
\text { superior }\end{array}$ & & \\
\hline Sangre de drago $100 \%$ & 15 & 10,80 & 0,775 & 0,200 & 10,37 & 11,23 & 10 & 12 \\
\hline Sangre de drago $75 \%$ & 15 & 8,20 & 0,414 & 0,107 & 7,97 & 8,43 & 8 & 9 \\
\hline Sangre de drago $50 \%$ & 15 & 6,00 & 0,00 & 0,00 & 6,00 & 6,00 & 6 & 6 \\
\hline Clorhexidina (Control Positivo) & 15 & 23,07 & 0,961 & 0,248 & 22,53 & 23,60 & 22 & 24 \\
\hline Agua (Control Negativo) & 15 & 6,00 & 0,00 & 0,00 & 6,00 & 6,00 & 6 & 6 \\
\hline Total & 75 & 10,81 & 6,445 & 0,744 & 9,33 & 12,30 & 6 & 24 \\
\hline
\end{tabular}

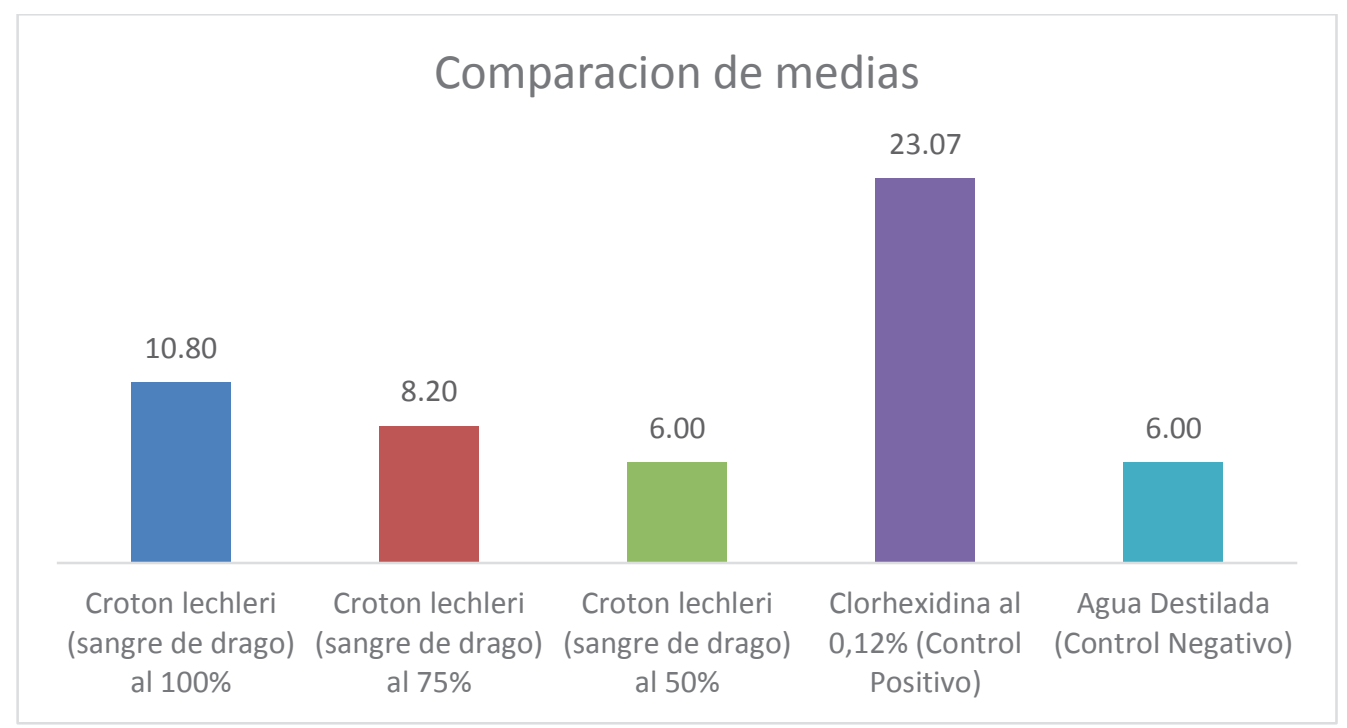

Figura 2. Comparación de medidas de los halos inhibitorios 
tes en la cavidad bucal, como Streptococcus agalactiae, Streptococcus uberis, Strepcoccus sanguis y Staphylococcus aureus.

Los resultados mencionados se correlacionan con esta investigación teniendo en cuenta que el $S$. mutans es un microorganismo característico de la cavidad bucal, Gram positivo, catalasa negativo, anaerobio facultativo, en agar sangre se distinguen colonias ordenadas en parejas y cadenas, altas, convexas, en forma de cojín, mucoide, de 0,5 a $1 \mathrm{~mm}$ de diámetro y opacas ${ }^{8}$.

El estudio utilizó a $C$. lechleri en tres concentraciones diferentes: 50, 75 y 100\% desarrollando halos de inhibición con promedios de 6,8 y $11 \mathrm{~mm}$ respectivamente sobre cepas de $S$. mutans. Estos resultados coindicen con los presentados por Cayo et al. ${ }^{17}$, cuyo estudio se realizó con distintas concentraciones de $C$. lechleri, originaria de Perú, obteniendo actividad antibacteriana in vitro sobre cepas de S. mutans. En la concentración al 40\% utilizada por Cayo et $a l^{17}$, se observaron halos de inhibición mínimos mientras que la concentración al $50 \%$ no obtuvo actividad alguna, sin embargo, esta diferencia no es significativa debido a que en ambos casos la bacteria no fue sensible. Además, cabe mencionar que en el estudio realizado, se comparó con un agente antibacteriano muy utilizado a nivel bucal como es la clorhexidina al $0,12 \%$, diferenciándose de esta manera del estudio mencionado.

Según Lazo et al. ${ }^{22}$, C. lechleri tiene la capacidad de disminuir en cierto modo la sobrepoblación bacteriana patógena de cavidad bucal, reduciendo de esta forma la sintomatología relacionada con la gingivitis del embarazo. De acuerdo con el estudio mencionado se determinó la actividad antibacteriana sobre $S$. mutans, colonizador nativo del medio bucal, por acción de $C$. lechleri en distintas concentraciones.

La sangre de drago posee en su composición ácido salicílico, el cual podría tener interacciones farmacológicas con trombolíticos y antiagregantes ${ }^{23}$, además de los flavonoides, cuya ingesta desmedida podría presentar sobreproducción de especies reactivas de oxígeno (ERO) y daños al ADN como a su proceso de replicación, los cuales pueden verse exacerbados durante el desarrollo fetal incrementando la sensibilidad a estos compuestos ${ }^{24}$.

En estudios realizados por Cevallos et al. ${ }^{25}$, se evaluó la toxicidad dérmica de C. lechleri aplicando una dosis de $2000 \mathrm{mg} / \mathrm{kg}$ en ratas Wistar, concluyendo que dichas dosis no producen toxicidad aguda dérmica, además no se observaron cambios de peso, daño en mucosa, ojos, ni el en sistema respiratorio, circulatorio, nervioso central y autónomo, en la actividad somatomotora y en los patrones de comportamiento a los 14 días de inspección.

Además de las características antibacterianas sobre cepas bacterianas de cavidad bucal, se ha promovido otro tipo de propiedades de este compuesto como lo aseveran estudios in vitro realizados por Risco et al. ${ }^{26}$; en cuyas investigaciones demuestran que la sangre de dra- go posee un efecto inmunomodulador, con capacidad para eliminar los radicales libres y dependiendo de la concentración puede poseer propiedades antioxidantes y pro oxidantes; a su vez la capacidad antiinflamatoria es una de las principales características de este compuesto natural.

La sangre de drago ha demostrado una capacidad de inhibición en la vía clásica y alternativa del sistema de complemento, así como también de la proliferación linfocitaria estimulada por mitosis con concavalina A y el crecimiento celular en leucemia linfoide, lo cual resulta en la capacidad de inhibición de las respuestas inmunes mediadas por células, que se puede atribuir a su componente 3',4-O-Dimethylcedrusin, el mismo que posee actividad potencialmente anti proliferativa y antitumoral ${ }^{26}$.

Sus propiedades antiinflamatorias significativas en la reducción de edema o formación de granulomas se deben al contenido del alcaloide taspina. El efecto antiinflamatorio se debe a la inhibición de la inflamación neurogénica al lograr un bloqueo en la activación de los nervios aferentes sensitivos a nivel pre y post sináptico ${ }^{27}$. Se ha descrito también la capacidad de la sangre de drago de estimular la fagocitosis en neutrófilos y monocitos humanos de una manera dosis dependiente en un rango de $5 \mu \mathrm{g} / \mathrm{mL}$ a $20 \mu \mathrm{g} / \mathrm{mL}^{26}$.

Las características cicatrizantes de la sangre de drago han sido ampliamente documentadas en la literatura, sin embargo, existe muy poca evidencia científica de su efecto antimicrobiano en el medio bucal. Por tanto, se tuvieron limitaciones de tipo bibliográfico debido a la poca evidencia científica existente en este campo, siendo el primer estudio realizado en el Ecuador para determinar la actividad antibacteriana de C. lechleri y necesitando nuevos estudios que ayuden a confirmar las propiedades antibacterianas de la sangre de drago.

Cabe mencionar que el estudio fue realizado con $C$. lechleri como se obtiene en estado natural, para futuras investigaciones se plantea la posibilidad de separar los distintos componentes por procedimientos como cromatografía para aprovechar de forma independiente sus propiedades, se sugiere estudios que analicen las características inmunológicas de la sangre de drago.

La prueba estadística de comparación por pares de Mann-Whitney determinó que la clorhexidina al 0,12\% produjo una mayor actividad antibacteriana que $C$. lechleri. Sin embargo, cabe mencionar que la actividad del látex, excepto la concentración al 50\%, es aceptable para tenerlo en cuenta como agente inhibitorio accesible y asequible frente al $S$. mutans.

Se concluye que las concentraciones al 75 y $100 \%$ de C. lechleri presentan una actividad antibacteriana sobre cepas de S. mutans, mientras que al 50\% de C. lechleri, el microorganismo presenta resistencia. La clorhexidina al 0,12\% como control positivo demostró mayor actividad antibacteriana que la sangre de drago, pero puede ser una alternativa natural, accesible y de bajo costo para métodos de prevención en caries dental. 


\section{Referencias bibliográficas}

1. Aguilera M, Romano E, Ramos N, Rojas L. Sensibilidad del Streptococcus mutans a tres enjuagues bucales comerciales. Odous Cient. 2011;12(1): 7-12.

2. Rojas S, Echeverría S. Caries Temprana de Infancia: ¿Enfermedad Infecciosas? Rev Med Clin Condes. 2014; 25(3):581-587.

3. Gonzáles S, Pedroso L, Rivero M, Reyes O. Epidemiología de la caries dental en la población venezolana menor de 19 años. Rev Cienc Méd La Habana. 2014;20(2):208-218.

4. MSP. Plan Nacional de Salud Bucal. [Consultado el 21 de enero 2018]. Accesible en: https://aplicaciones.msp. gob.ec/salud/archivosdigitales/documentosDirecciones/ dnn/archivos/PLAN\%20NACIONAL\%20DE\%20 SALUD\%20BUCAL.pdf.

5. MSP. Caries: Guía de Prácticas Clínicas. [consultado el 8 de diciembre 2015]. Accesible en: http://www.salud. gob.ec/wp-content/uploads/2014/05/CARIES.pdf.

6. Nuñez P, García L. Bioquímica de la caries dental. Rev Habanera Cienc Méd. 2010;9(2):156-166.

7. Fejerskov O, Kidd E. Dental Caries The desase and its Clinical Management. Second Edition ed. Singapore: Blackwell Munksgaard; 2008.

8. Gamboa F. Identificación y caracterización microbiológica, fenotípica y genotípica del Streptococcus mutans: experiencias de investigación. Univ Odontol. 2014;33(71):65-63.

9. Ojeda J, Oviedo E, Salas L. Streptococcus mutans and dental caries. Rev Ces Odont. 2013;26(1):44-56.

10. Moye Z, Zeng L, Burne R. Fueling the caries procces: carbohydrate metabolism and gene regulation by Streptococcus mutans. J Oral Microbiol. 2014;6. doi: 10.3402/ jom.v6.24878.

11. OPS. La Salud Oral de los Niños de Bajos Ingresos: Procedimiento para el Tratamiento Restaurativo atraumatico (PRAT). Informe Final. Washington, DC: OPS/ OMS. [Consultado el 20 de abril 2018]. Accesible en: http://new.paho.org/hq/dmdocuments/2009/OHPRAT_mar2009.pdf

12. Cuadrado D, Peńa R, JF. G. El concepto de caries: hacia un tratamiento no invasivo. Revista ADM. 2013;70(2):54-60.

13. Flores M, Vízques J, Rodríguez J. Efectividad del Theobroma cacao sobre el desarrollo del biofilm dental. Rev Cient Odontol. 2016;12(1):8-13.

14. Azevedo A, Pacheco S, García C. Efeito leishmanicida in vitro do látex de Croton lechleri (Euphorbiaceae). Rev Cienc Farm Básica Apl. 2015;36(3):413-418.
15. Corrales L, Castillo A, Melo A. Evaluación del potencial antibacterial in vitro del Croton Leclheri frente a asilamientos bacterianos de pacientes con úlceras cutáneas. NOVA. 2013;11(19):51-63.

16. Castillo-Quiliano A, Dominguez-Torrejon G. Evaluación de la producción de látex de sangre de drago (Croton lechleri) en función al diámetro y cuatro periodos de precipitación en poblaciones naturales en Ucayali, Perú. Ecol apl. 2010;9(2):61-69.

17. Cayo C, Barrera R. Evaluación in vitro del efecto antibacteriano del Croton lechleri sobre cultivos de Streptococcus mutans (ATCC 25175). Cienc Desar. 2014; 17(1):5-10.

18. Tamariz J, Capcha R, Palomino E, Aguilar J. Actividad antibacteriana de la Sangre de Grado (Croton lechleri) frente a Helicobacter Pylori. Rev Med Hered. 2003;14(2):81-88.

19. Gallardo G, Barboza L. Efecto cicatrizante del gel elaborado del látex de Croton Lechleri "Sangre de Drago". Rev Cient Cienc Med. 2015;18(1):10-16.

20. OPS. Manual de Pruebas de Susceptibilidad Antimicrobiana. [Consultado el 13 de febrero del 2018] .Accesible en: http://www.paho.org/hq/index.php?option=com_ docman \&task=doc_view \&gid=22691\&Itemi$\mathrm{d}=1639$ \&lang=en.

21. León K, Santiago J. Propiedades antimicrobianas de películas de Quitosano-Alcohol polivinílico embebidas en extracto de sangre de drago. Rev Soc Quím Perú. 2007;73(3):158-165.

22. Lazo J, Pareja M. Extracto de Croto lechleri y de Pelargonium Robertianum L. en el tratamiento de la gingivitis asociada al embarazo. Kiru. 2007;4(2):52-59.

23. Tres J. Interaccion entre farmacos y plantas medicinales. An Sist Sanit Navar. 2006;29(2):233-252.

24. Estrada Reyes R, Ubaldo Suarez D, Araujo Escalona A. Los flavonoides y el Sistema Nervioso Central. Salud Ment. 2012;35(5):375-384

25. Cevallos D, Jaramillo C, Cuesta O, Cuesta O, Zaldua J, García G, et al. Composición química, actividad cicatrizante y toxicidad del látex Croton lechleri. Rev Cient. 2016;26(2):95-103.

26. Risco E, Ghia F, Vila R, Iglesias J, E A, Cañigueral S. Immunomodulatory Activity and Chemical Characterisation of Sangre de Drago from Croton lechleri. Planta Med. 2003;69:785-794.

27. Gupta D, Bleakley B, R. G. Dragon's blood: Botany, chemistry and therapeutic uses. J. Ethnopharmacol. 2007;115(3):361-380. 